\title{
IMPACTO DO FUNDO DE PARTICIPAÇÃO DOS MUNICÍPIOS (FPM) SOBRE A QUALIDADE DA SAÚDE DOS MUNICÍPIOS BRASILEIROS
}

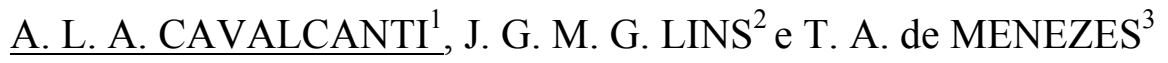 \\ ${ }^{1}$ Universidade Federal de Pernambuco, Mestrando em Gestão e economia da Saúde. \\ ${ }^{2}$ Universidade Federal de Pernambuco, Doutorando em Economia. \\ ${ }^{3}$ Universidade Federal de Pernambuco, Doutora em Economia pela Universidade de São \\ Paulo (USP) e Professora Associada do Departamento de Economia da UFPE. \\ E-mail para contato: Andre_luizcavalcanti@hotmail.com
}

\begin{abstract}
RESUMO - Embora o Sistema Único de Saúde (SUS) garanta o acesso universal e uma assistência integral, muito estudos sugerem que o seu financiamento não tem sido compativel para garantir tais princípios doutrinários. Diante dessa realidade, o objetivo do presente estudo foi verificar se o maior financiamento governamental, através do Fundo de Participação dos Municipios (FPM), afetou positivamente indicadores de saúde materna e infantil nos pequenos municípios do país no ano de 2015. A existência de descontinuidades nos aportes do FPM, em pontos de intervalos populacionais é usada no arcabouço metodológico para contornar problemas de verificação do efeito causal de interesse sem interferência de fatores confundidores, heterogeneidade local e variáveis explicativas de saúde não observáveis. Os resultados do estudo mostraram que choques positivos no financiamento do FPM, não se refletiram em uma melhora dos indicadores materno-infantil. Este é um fato importante, pois parece que outros fatores relevantes, como por exemplo, gestão, corrupção e pouca fiscalização dos gastos, podem estar interferindo drasticamente na eficácia desse financiamento.
\end{abstract}

Palavras-Chave: Economia da Saúde; Indicadores de Saúde; Eficiência dos Gastos Públicos.

ABSTRACT - Although Brazil's PublicUnified HealthSystem (SUS) guarantees universal access and comprehensive care, many studies suggest that its financing is not compatible to guarantee such doctrinal principles. In face of this reality, the objective of the present study was to identify if the increased governmental funding, through the Municipal Participation Fund (FPM), has affected positively maternal and child health indicators in the small municipalities of the country in the year 2015. The existence of discontinuities in the FPM contributions, in population ranges, is applied to the methodological frameworkin order to overcome problems with the selection of the causal effect of interest without the interference of confounding factors, local heterogeneity and unobservable explanatory variables of health. Thus, we present a quantitative study using a methodology that compares the means of the variables of interest between the treated group (municipalities receiving more federal funding) and the control group (municipalities receiving less federal funding). The results of the study show that positive impacts in FPM fundingdid not reflect an improvement in maternal and child indicators. 
This is an important fact, because it seems that other relevant factors, such as management, corruption and poor expenditures control, may be drastically interfering inthe effectiveness of this funding.

Keywords: Health Economics; Health Indicators; Efficiency of Public Expenditures.

\section{INTRODUÇÃO}

Embora o Sistema Único de Saúde (SUS) garanta o acesso universal e uma assistência integral, o seu financiamento não tem sido compatível para garantir tais princípios doutrinários (MARQUES; MENDES, 2012). Ainda de acordo com o Instituto de Pesquisa Econômica Aplicada (IPEA) o gasto público per capita com saúde do Brasil é de quatro a sete vezes menores do que o de países que apresentam sistema universal de saúde, como Reino Unido e França, e ainda inferior aos países da América do Sul que não garante acesso universal a tais serviços. (VIEIRA; BENEVIDES, 2016). Atrelado a isso, a eficiência dos gastos públicos em saúde, tem se tornado alvo de discussão nos principais congressos nacionais de saúde pública e estudos nessa área, tem despertado sobre a importância da alocação dos recursos de forma eficiente.

Diante do processo de financiamento das políticas pública em saúde e a qualidade dos seus indicadores nos territórios brasileiros é possível questionar se maiores transferências intergovernamentais em saúde, em especial o Fundo de Participação dos Municípios (FPM), de fato, estão associados a melhores indicadores de saúde. Neste contexto, a avaliação em economia da saúde tem se tornado uma área de atuação cada vez mais importante para a institucionalização da economia da saúde no SUS, bem como, subsidiar os gestores locais no processo de decisão de políticas públicas que permitam uma melhor efetividade nas ações e serviços de saúde (BRASIL, 2012). Porém, estudos dessa natureza, são de difíceis realizações, tendo em vista os fatores determinantes e condicionantes de saúde, previsto na Lei de $n^{\circ} 8.080$ de 19 de setembro de 1990, no qual estão imbricados aos diversos problemas de saúde existentes no Brasil.

Tendo isso em vista, o objetivo deste trabalho foi de verificar como choques positivos no financiamento federal aos municípios se refletem em uma melhora de alguns indicadores de saúde materno-infantil. Por causa de inúmeros elementos confundidores (como desigualdades socioeconômicas, diferenças culturais, etc.) que afetam as variáveis de saúde coletiva, a verificação desse efeito de interesse é de difícil execução e, por isso, verificamos que seus resultados, na maioria dos trabalhos apresentados, tendem a levar a interpretações errôneas. O principal motivo para isso é devido ao fato que estas pesquisas geram confusão entre o que é uma associação de mera correlação entre variáveis e o que pode ser inferido como um efeito causal sem problemas de interferência desses outros importantes determinantes. A utilização dos choques de financiamento baseados nas regras do FPM já foi usada na literatura internacional ${ }^{1}$. Como será detalhado na estratégia empírica, a principal vantagem dessa metodologia é que ela permitir emular um quase experimento aleatório, gerando uma divisão e comparação de dois grupos (Tratado e Controle), de modo que a estratégia é capaz de expurgar os fatores endógenos aos municípios que podem contaminar a relação de causa e efeito de interesse.

Por fim, a principal justificativa para este trabalho é que estudos dessa natureza podem gerar diversos subsídios para elaboração de políticas públicas em saúde mais

\footnotetext{
${ }^{1}$ Por exemplo, Brollo et al. (2013), ao utilizarem-se desse mecanismo de financiamento, verificaram os efeitos das receitas adicionais do governo sobre a corrupção política e a qualidade dos políticos através da utilização de um Desenho de Regressão Descontínua.
} 
eficientes, principalmente ao reforçar ou refutar hipóteses ligadas à relação entre maiores financiamentos governamentais e a melhor qualidade de indicadores locais de saúde; notadamente, no atual cenário, em que o tema de contingenciamento dos gastos em saúde tem se destacado.

\section{ESTRATÉGIA EMPÍRICA}

Para contornar problemas oriundos de elementos confundidores que dificultam a análise de causa e efeito de nosso interesse, utilizamos uma estratégia empírica baseada na existência de descontinuidades presente na regra de financiamento do FPM ${ }^{2}$. Neste trabalho, exploramos a possibilidade de existência de descontinuidades geradas nas médias dos indicadores de saúde materna e infantil a partir das descontinuidades do financiamento do FPM, oriundos dos cortes populacionais entre os diferentes grupos que o compõe. Essa estratégia empírica baseia-se na hipótese de que há uma relação contínua entre a variável de seleção (a população total do município do último Censo demográfico, realizado em 2010) e os indicadores de saúde e, por conseguinte, qualquer descontinuidade na função condicional dessa variável de interesse pode ser atribuída ao salto que ocorre no financiamento municipal em torno dos cortes populacionais estabelecidos na regra do FPM.

Assim, foi possível verificarmos como choques adicionais de receita federal em ações de saúde afetaram os indicadores de interesse, comparando municípios imediatamente acima de determinado cutoff populacional (grupo tratado e que recebe mais financiamento) com municípios imediatamente abaixo do mesmo cutoff populacional (grupo controle e que recebe menos financiamento) estabelecidos pela regra de aporte do FPM. De modo que, partindo da hipótese de exogeneidade de cada cutoff, a estratégia empírica permitiu controlar para possíveis problemas de fatores diversos, nos garantindo choques exógenos de receitas federais (livres da interferência de outros determinantes) e suas implicações na média dos indicadores de saúde local.

Devido à grande heterogeneidade social e econômica entre os municípios do Brasil, essa estratégia empírica verificou o efeito do choque de financiamento apenas nos pequenos municípios do país, com população de até 50.940 mil habitantes. Esta abordagem tem uma importante justificativa: os municípios pequenos, em geral, tendem a ter baixa capacidade de arrecadação tributária, dependendo fundamentalmente de receitas intergovernamentais. Estes municípios, no geral, apresentam um alto grau de dependência das outras esferas do governo quanto ao financiamento dos seus serviços públicos de saúde e dispõem de menos opções de arrecadação de recursos próprios para destinar a essa área. Este fato é essencial, pois um choque adicional de verba tende a ter um efeito mais forte neste tipo de localidade (BROLLO et al., 2013). Ao incluir médios e grandes municípios na amostra, que tendem a possuir maior capacidade de arrecadação tributária e possibilidade de aplicar uma maior quantidade de recursos próprios na saúde, poderíamos estar mascarando o efeito do financiamento federal sobre os indicadores de saúde.

Os indicadores das variáveis independentes (FPM) e dependentes (taxa de mortalidade infantil, taxa de mortalidade materna e de consultas pré-natal) foram coletados através de site de domínio público, disponíveis pelo Tribunal de Contas da União (TCU) e pelo Ministério da Saúde/DATASUS, respectivamente, e posteriormente tabulados no excel. Para aplicação do tratamento estatístico utilizou-se o software STATA versão 14.0.

\footnotetext{
${ }^{2}$ Ver figura 01
} 


\section{RESULTADOS E DISCUSSÃO}

O estudo se propôs a avaliar o impacto da transferência constitucional intergovernamental do FPM nos indicadores de saúde, mais detalhadamente sobre as Taxa de Mortalidade Infantil (TMI), Taxa de Mortalidade materna (TMM) e Taxa de Consultas Pré-Natais. Como já destacado, a pergunta ser respondida é se um choque adicional dessa verba poderia impactar na qualidade de indicadores de saúde, a partir da comparação das médias dos indicadores de interesse nos grupos tratados e controle.

Figura 01. Transferências totais do Fundo de Participação dos Municípios do Brasil em 2015 entre os diferentes intervalos de financiamento (valores em unidades de R\$ 100 mil).

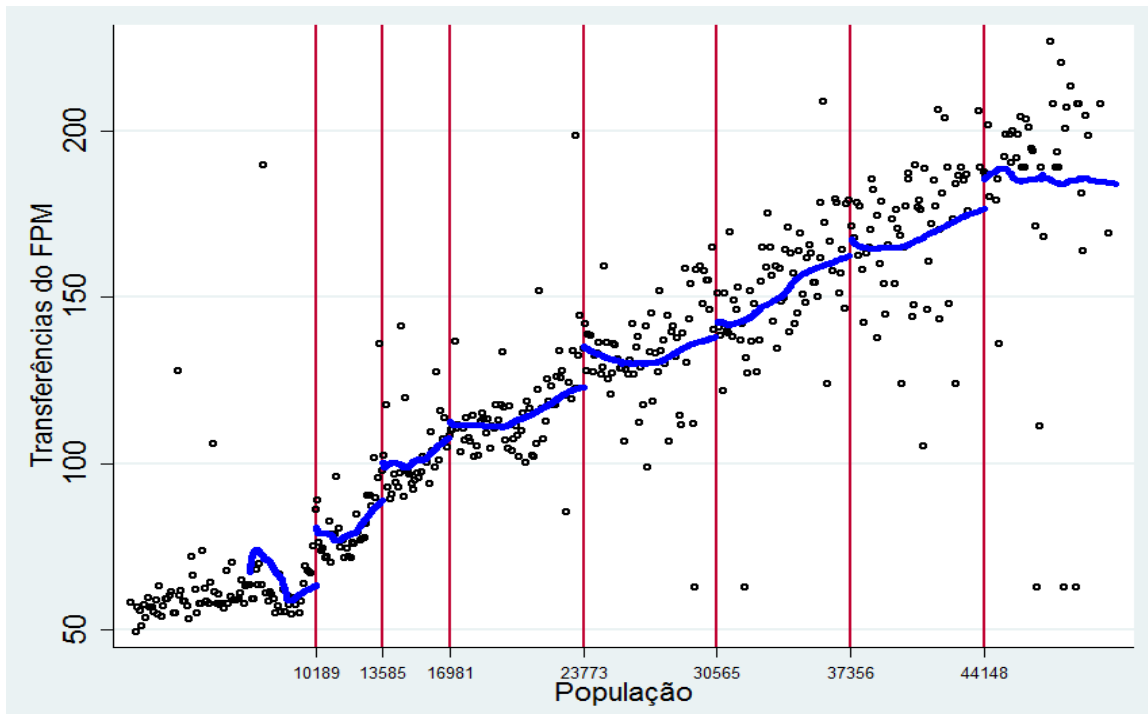

Fonte: Elaboração própria com base nos dados do Tribunal de Contas da União (TCU).

A figura 01 mostra que de fato existe um choque de financiamento nas descontinuidades do repasse do FPM a partir dos cortes populacionais adotados pelo TCU, sendo estes, o nosso instrumento exógeno que aloca os municípios brasileiros, quase que aleatoriamente, em intervalos populacionais previamente estabelecidos. Observando detalhadamente a figura 01 é possível verificar a média (estimada por uma função kernel) do repasse federal em cada intervalo populacional, bem como os saltos nessa média a partir de cada corte. Vale reforçar que o estudo se propôs a verificar a média das variáveis de interesse a partir de todos os cortes adotados no estudo, e não apenas, onde a descontinuidade nos valores do repasse do FPM é maior ou menor. Nesse caso, estamos interessados no efeito global do repasse nos pequenos municípios, uma vez que uma análise em cada um dos cortes nos daria apenas, o efeito local, restrito aquela região populacional.

A figura 2 apresenta o efeito do repasse do FPM sobre os indicadores de saúde, entre os grupos, a nível de Brasil, os resultados mostraram-se diversos. No caso da mortalidade infantil não foi encontrado nenhuma diferença visível da média entre os dois grupos. No caso de mortalidade materna, os municípios tratados apresentaram leve queda na média desse indicador. No caso de consultas pré-natais parece não haver diferença visível no caso de nenhuma consulta. Por outros lado, os municípios que receberam mais financiamento apresentaram queda nos indicadores de até 6 consultas e elevação da média no caso de mais de 7 consultas. Assim, parece que, pelo menos nessa análise preliminar, os municípios que recebem maiores repasse do FPM apresentaram melhores indicadores de mortalidade materna e consultas pré-natal. 
Figura 2. Efeito dos indicadores de saúde entre os grupos tratados e controle.
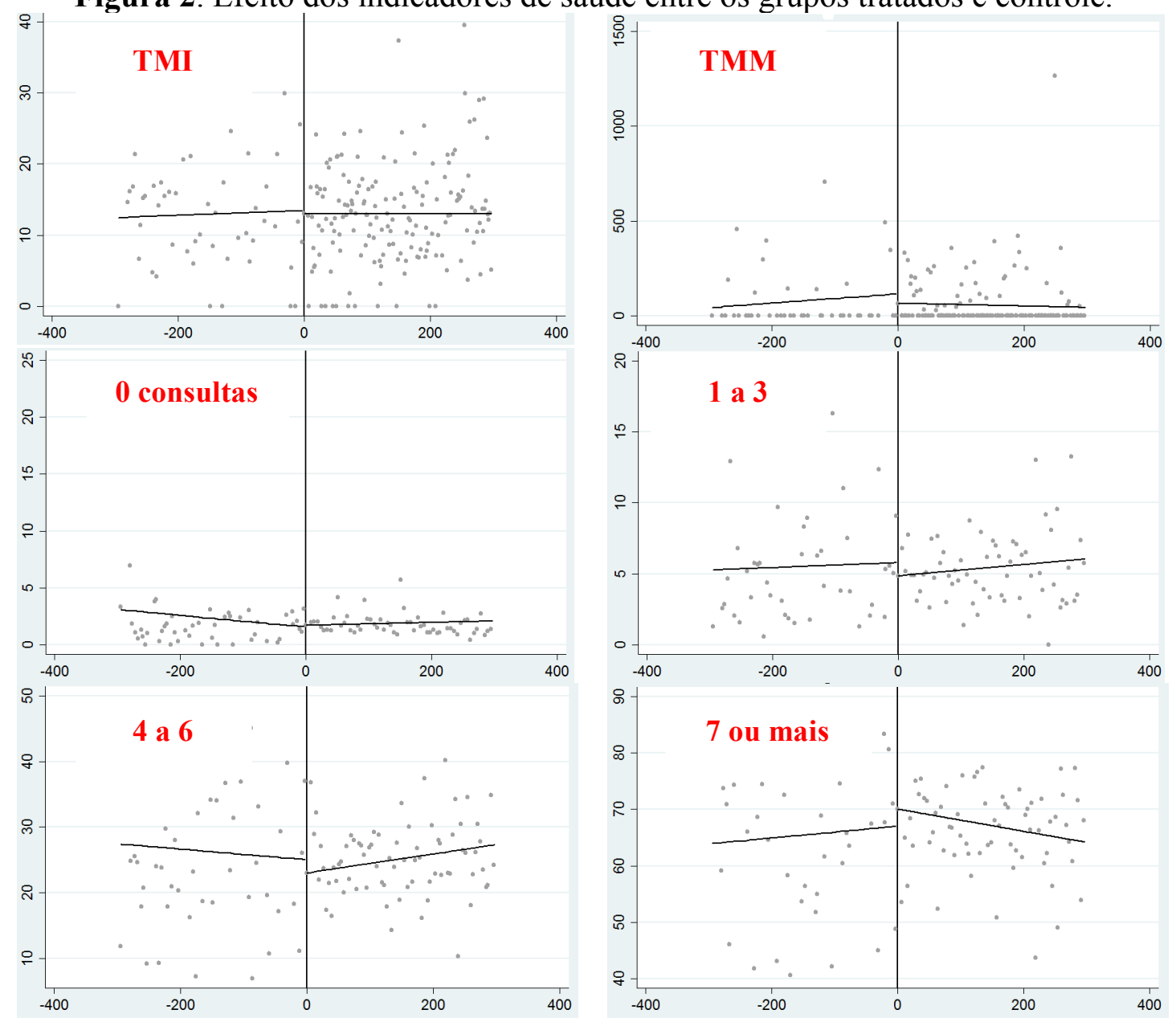

Fonte: Elaboração própria com base nos dados do Ministério da Saúde.

Embora esses resultados nos dêem uma análise preliminar, é preciso que eles sejam avaliados mediante testes de inferência que nos digam se essas possíveis diferenças de média são estatisticamente significativas. Assim, como abordagem complementar, verificamos através de uma regressão descontínua ${ }^{3}$, se houve uma diferença significativa dos indicadores do grupo tratado em relação ao grupo controle dentro de uma banda populacional de 300 habitantes em relação a cada cutoff definido pela regra de financiamento do FPM. Os resultados apresentados na tabelas 1 mostram que não foi encontrada nenhuma significância nos parâmetros estimados, ou seja, estatisticamente não há diferença alguma dos indicadores entre os dois grupos.

Tabela 1. Resultado da regressão descontínua com o uso dos indicadores de saúde (bandwidth de 300 habitantes).

\begin{tabular}{cccc}
\hline Variáveis & Coeficiente & Erro-padrão & p-valor \\
\hline Tx. Mortalidade Infantil & $-0,5789$ & 3,8265 & 0,88 \\
Tx. Mortalidade Materna & $-51,289$ & 76,89 & 0,50 \\
Tx de nenhuma consulta pré-natal & 0,3394 & 0,3215 & 0,29 \\
Tx de 1 a 3 consultas pré-natal & 0,1887 & 0,6443 & 0,70
\end{tabular}

\footnotetext{
${ }^{3}$ Para mais detalhes desse procedimento estatístico ver Cattaneo et al. (2016).
} 
Tx de 4 a 6 consultas pré-natal

Tx de 7 ou mais consultas pré-natal
2,0504

0,93

0,2963

2,5591

0,90

realizada com efeito fixo de região e os erros-padrão robustos em cluster de município.

O estudo de Simão e Orellano (2015), investigou a existência de uma relação positiva entre receita municipal per capita, incluindo os repasses constitucionais intergovernamentais, e as transferências do SUS fundo a fundo. A partir das análises, os resultados mostraram que de fato existe uma correlação positiva, onde os municípios que apresentam maiores receitas per capita, recebem mais dinheiro de transferência do SUS fundo a fundo, através do $\mathrm{PAB}$ variável, ao qual está associado à adesão de novos serviços e ou programas do SUS, justificando que estes municípios apresentam maiores capacidades financeiras de manterem os custos adicionais de tais programa/serviços.

Outro ponto bastante importante e que foi avaliado nesse mesmo estudo é que, essa relação positiva entre, maiores receitas municipais per capita e maiores incentivos de transferências do SUS, avaliando no aspecto redistributivo, pode não ser desejada, em detrimento de que, esses incentivos de transferências do SUS para municípios com maiores receitas, não acontecem de forma criteriosa, como por exemplo a partir da eficiência dos gastos públicos em saúde.

Essa realidade pode ser corroborada no estudo de Andrett e Rosa (2015), que se utilizando da metodologia da Análise Envoltória de Dados (DEA), verificou a eficiência dos gastos públicos em saúde nas 27 unidades de federação brasileira, entre os anos de 2005 a 2014, através dos gastos per capita de despesa com saúde. Os resultados do estudo apontaram que apenas 09 das 27 unidades de federação, apresentaram 100\% eficientes em pelo menos um ano, demonstrando que de fato existe uma ineficiência dos gastos públicos em saúde.

Por fim, o estudo de Menezes (2017) questiona se a estratégia das transferências intergovernamentais, através de descentralização de receitas governamentais, melhora o crescimento econômico, saúde e educação. Os resultados mostraram dois pontos importantes: o primeiro é que maiores recursos de descentralização induzem mais crescimento do PIB per capita e reduzem o desempenho da saúde e da educação, podendo justificar a não diferença entre os grupos do presente estudo, sobre os indicadores de saúde. O segundo ponto é que os municípios que sofreram punição da Controladoria Geral da União, antes da eleição, apresentaram melhor crescimento per capita e o desempenho da saúde em comparação com os municípios auditados após a eleição, demonstrando que existe um efeito muito forte da corrupção sobre a eficiência dos gastos públicos.

\section{CONCLUSÃO}

Os resultados do presente estudo mostraram que não houve nenhuma diferença estatística dos indicadores de saúde, entre os grupos tratado e controle, o que sugere que maiores repasses do FPM não afetou as ações de saúde destas pequenas localidades, pelo menos no que diz respeito a esses indicadores, no ano de 2015.

A partir dos resultados é possível concluir que, maiores investimentos em saúde, não necessariamente implicarão em melhores resultados e que fatores ligados a corrupção podem ser um dos problemas que contribuam para uma má administração desses recursos. Para tanto, estudos mais aprofundados e que se utilizem de estratégicas empíricas que permitam verificar o controle da aplicação destes recursos, possam dar continuidade a pergunta norteadora do presente estudo de forma mais completa. Assim, a fim de verificar melhor os efeitos das transferências constitucionais sobre a qualidade da saúde dos municípios brasileiros, é necessário incluir na análise como a governança institucional 
pode interferir na aplicação destes recursos e, portanto, contribuir para a uma maior eficiência nos gastos públicos.

\section{REFERÊNCIAS}

ANDRETT, M. C. S.; ROSA, F. S. Eficiência dos gastos públicos em saúde no Brasil: estudo sobre o desempenho de estados brasileiros. In: CONGRESSO BRASILEIRO DE CUSTOS, 22.,2015, Foz do Iguaçu-PR. Anais Eletrônicos....Foz de Iguaçu: Associação Brasileira de

Custos, 2015. Disponível: <https://anaiscbc.emnuvens.com.br/anais/index >. Acesso em 08/10/2017.

BRASIL. Ministério da Saúde. Secretaria-Executiva/Secretaria de Atenção à Saúde: Glossário temático: economia da saúde / Ministério da Saúde, Secretaria-Executiva, Departamento de Economia da Saúde, Investimentos e Desenvolvimento. - 3. ed.Brasília: Ministério da Saúde, 2012. Disponível em: $<$ http://bvsms.saude.gov.br/bvs/publicacoes/comites_mortalidade_materna_3ed.pd f>. Acesso em: 14 Nov. 2016.

BROLlO F. et al. The Political Resource Curse .American Economic Review, v. 105, n. 5, p.1759-1796, 2013.

CATTANEO, M. D. et al. Interpreting regression discontinuity designs with multiple cutoffs. The Journal of Politics, v. 78, n. 4, p. 1229-1248, 2016.

HSIAO, W. C. Why Is A Systemic View Of Health Financing Necessary? Health Affairs, v. 26, n. 4, 2007.

MARZON, L. M.; MASCARENHAS, L. P. G.; DELLABRIDA, V. R. Eficiência dos Gastos Público em Saúde: Desafio para municípios de Santa Catarina, Brasil. Saúde Sociedade. São Paulo, v.24, n.1, p.23-33, 2015.

MARQUES, R. M.; MENDES, A. A problemática do financiamento da saúde pública brasileira: de 1985 a 2008. Economia e Sociedade, Campinas, v. 21, n. 2 (45), p. 345$362,2012$.

MENEZES, T. A. The Impact of Decentralization Revenue on Economic Growth and Wellbeing.in: I CONGRESS LATIN AMERICAN AND CARIBBEAN REGIONAL SCIENC ASSOCIATION INTERNATIONAL/ XV ENCONTRO NACIONAL DA ASSOCIAÇÃO BRASILEIRA DE ESTUDOS REGIONAIS E URBANOS, São Paulo, anais... São Paulo: ENABER, 2017.

SIMÃO, J. B.; ORELLANO, V. I. F. Um estudo sobre a distribuição das transferências para o setor de saúde no Brasil. Estud. Econ., São Paulo, v. 45, n. 1, 2015.

VIEIRA, F. S.; BENEVIDES, R. P. S. (Org). Os impactos do novo regime fiscal para o financiamento do sistema único de saúde e para a efetivação do direito à saúde no brasil. / Nota Técnica $n^{\circ}$ 28. Brasília, DF: IPEA, 2016. 


\section{AGRADECIMENTOS}

Ao DESiD - Ministério da Saúde e a Universidade Federal de Pernambuco pela idealização e concretização do Mestrado profissional em Gestão e Economia da Saúde, possibilitando a qualificação de profissionais de saúde e gestores para o aperfeiçoamento dos serviços público de saúde do estado de Pernambuco. 